\title{
The levels of endothelial growth factor (VEGF) and its receptors in ovarian hemorrhages
}

\begin{abstract}
The morphological substrate of ovarian apoplexy is a cyst of the corpus luteum with haemorrhage (HLC) into the cyst cavity and rupture of the capsule $(88.15 \%)$, corpus luteum with haemorrhage (in 8.89\%), follicular cyst with haemorrhage (in $2.96 \%$ ). HLC found a significant increase of expression of VEGF and its receptors (VEGFR1, VEGFR2) in vascular endothelium and granular lutein cells of corpora lutea, a change in the VEGFR1: VEGFR2 expression ratio in the endothelium of corpora lutea as compared to the norm. A significant increase of the sizes of vessels, their area and different variants of vascular architectonics in OA was found.
\end{abstract}

Keywords: hemorrhagic corpus luteal cyst, Vascular Endothelial Growth Factor, pathogenetic mechanisms
Volume 3 Issue $3-2018$

\section{Davydov Al, Shahlamova MN}

Professor of medicine at the chair of obstetrics, gynaecology and perinatology, I.M.Sechenov First Moscow State Medical University, Russia

Correspondence: Davydov Aleksandr Il'gizirovich, Professor of medicine at the chair of obstetrics, gynaecology and perinatology, I.M.Sechenov First Moscow State Medical University, Russia, Email al-davydov@mail.ru

Received: February 16, 2018| Published: June 07, 2018

\section{Introduction}

Among the causes of intra-abdominal bleeding in young women, hemorrhagic corpus luteal cyst (HLC) occupies the second place after ectopic pregnancy. The source of bleeding is often a defect in the capsule of the cyst of yellow body, rarely it is the defect of yellow body, of follicular cysts and of ovarian tissue. A key role in the formation of the vascular network of the follicle and yellow body plays Vascular Endothelial Growth Factor (VEGF), which implements its effects through the first (VEGF1) and second (VEGF2) receptors. Both increased and reduced expression of VEGF lead to disruption of angiogenesis and vascular permeability with formation of ovarian cysts and with hemorrhage into the cavity. With all morphological variants of HLC there is hemorrhage in the ovarian tissue, however, causes and mechanisms of its occurrence are still poorly investigated. ${ }^{1,2}$

\section{Objective}

is to study the expression of VEGF, its receptors (VEGF1, VEGF2) and particularities of the vascular network of the cysts/yellow bodies in patients with HLC.

\section{Patients}

The study involved 152 patients (mean age of $24.13 \pm 0.33$ years) with the anemic form of the HLC. Fragments of the wall of cyst/ yellow body with antibodies to VEGF, VEGF1, VEGF2 and CD34 were studied. As a control served normal yellow bodies (the material of the pathological laboratory).

\section{Results}

Morphological study found hemorrhagic corpus luteal cyst with capsule rupture $(88,2 \%)$, yellow body with hemorrhage and destruction $(8,9 \%)$, follicular cyst with bleeding and rupture of the capsule $(2,96 \%)$. The degree of cystic transformation of yellow body at HLC varies from moderate enlargement of the gap of yellow body with conservation of a broad layer of granulosa-luteal cells before rapid thinning of the wall with preservation of a thin layer of theca luteal and granulosa-luteal cells.

In the vascular endothelium and granulosa-luteal cells of cysts/ yellow bodies in patients with HLC a significant increase in the expression of VEGF and its receptors, compared to normal yellow bodies, was found. Expression of VEGF2, which stimulated proliferative activity of endothelial cells, was higher than the expression of VEGF1, responsible for the formation of full vessels in both groups. At the same time, in patients with HLC the measure of the ratio of VEGF1/ VEGF2 in vascular endothelium was significantly higher $(0,51 \pm 0,05$ points $)$ compared with the control $(0,21 \pm 0,1$ points).

This indicates a more pronounced increase in the level of VEGF1 in vascular endothelium of yellow body with HLC and indicates the tendency to the formation of "mature" blood vessels on the background of relative decrease of proliferative activity of endothelial cells. The use of antibodies to CD34 allowed us to visualize the vasculature of yellow body with morphometric evaluation of its parameters. In normal yellow bodies numerous small thin-walled vessels, evenly spaced among granulosa cells, were defined.

Vasculature of yellow bodies at HLC was remarkable for significant increase in the size of vessels and their area. These parameters did not depend on the size of cysts/yellow bodies, but was significantly higher in patients with functional ovarian cysts in anamnesis. Architectonics of vessels at HLC ranged from normal to the formation of the large vessels of the sinusoidal type in the tech and granulate, prone to destruction. The detected peculiarities of vascularization of cysts/ yellow bodies were consistent with the results of a study of expression of VEGF and its receptors.

Retrospectively the average level of VEGF in the acute period of HLC and after 3, 6 months were not significantly different, which testified the local character of changes of angiogenesis in ovarian tissue. However, in $50 \%$ of patients in the acute period its level was increased. The concentration of VEGF in the blood plasma in the acute period of HLC was in inverse proportion to the levels of the contents of this growth factor in vascular endothelium $(\mathrm{r}=-0,78$, $\mathrm{p}=0,022$ ). Determination of the concentration of VEGF in plasma does not reflect the full rate of the changes in yellow body, but can indicate the difference of pathogenetic mechanisms of formation of the HLC (Figure 1). 


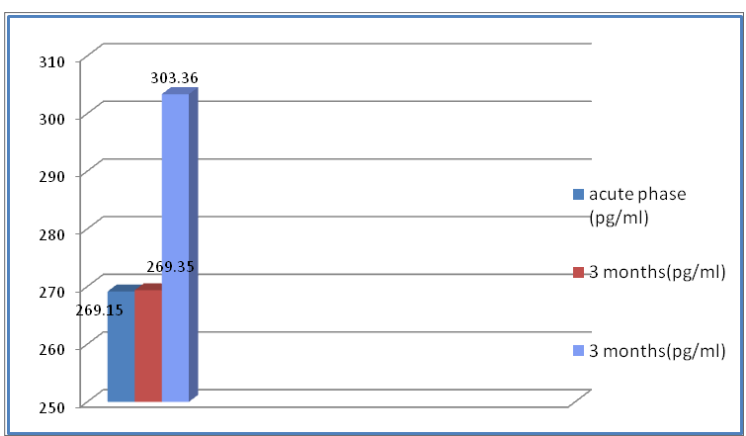

Figure I LevelVEGF in patients with HLC

\section{Conclusion}

Thus, one of the pathogenetic mechanisms of the formation of hemorrhage in the ovarian tissue at HLC are disorders of angiogenesis, in the development of which the growth of the expression of VEGF and its receptors in vascular endothelium and granulosa-luteal cells, as well as a violation of the ratio of VEGF1/ VEGF2 in the endothelium of cysts/yellow bodies, play an important role.

\section{Acknowledgements}

None.

\section{Conflict of interest}

The author declares no conflict of interest.

\section{References}

1. Trau HA, Davis JS, Duffy DM. Angiogenesis in the primate ovulatory follicle is stimulated by luteinizing hormone via prostaglandin E2. Biol Reprod. 2015;92(1):15.

2. Hall AP, Ashton S, Horner J, et al. PDGFR Inhibition Results in Pericyte Depletion and Hemorrhage into the Corpus Luteum of the Rat Ovary. Toxicol Pathol. 2016;44(1):98-111. 